\title{
Scanning Electron Microscopy of the Presbylarynx
}

\author{
Tatiana Maria Gonçalves, MD', Daniela Carvalho dos Santos, PhD², \\ Adriana Bueno Benito Pessin, PhD', and \\ Regina Helena Garcia Martins, MD, PhD'
}

Sponsorships or competing interests that may be relevant to content are disclosed at the end of this article.

\begin{abstract}
Objective. To describe the findings on the presbylarynx under scanning electron microscopy.

Study Design. Cadaver study.

Setting. Universidade Estadual Paulista (Botucatu, São Paulo, Brazil).

Subjects and Methods. Sixteen vocal folds were removed during necropsies and distributed into 2 age groups: control $(\mathrm{n}=8$; aged 30-50 years) and elderly $(\mathrm{n}=8$; aged 75-92 years). The right vocal fold was dissected, fixed in glutaraldehyde $2.5 \%$, and prepared for scanning electron microscopy. The thickness of the epithelium was measured using a scandium morphometric digital program.
\end{abstract}

Results. In the control group, the epithelium had 5 to 7 overlapped cell layers, rare desquamation cells, and little undulation with protruding intercellular junctions. The lamina propria showed a uniform network of collagen and elastic fibers in the superficial layer. A dense network of collagen was identified in the deeper layer. In the elderly group, the epithelium was atrophic (2-3 cells), with more desquamation cells and intercellular junctions delimited by deep sulci. The epithelial thickness was lower in elderly than in controls (mean [SD], 221.64 [145.90] $\mu \mathrm{m}$ vs 41.79 [21.40] $\mu \mathrm{m}$, respectively). The lamina propria had a dense and irregular distribution of collagen and elastic fibers in the superficial layer. In the deep layers, the collagen fibers formed a true fibrotic and rigid skeleton.

Conclusion. Scanning electron microscopy identified several changes in the elderly larynx, differentiating it from the controls. These alterations are probably related to the aging process of the vocal folds. However, the exact interpretation of these findings requires additional studies, even to the molecular level, having the fibroblasts as targets.

\section{Keywords}

presbylarynx, scanning electron microscopy, voice, presbyphonia, dysphonia, elderly

Received September 15, 2015; revised February 9, 2016; accepted February 12, 2016.
$\mathrm{P}$ resbyphonia is a set of vocal changes secondary to aging of laryngeal structures. ${ }^{1}$ Under such conditions, the aged voice acquires some peculiar features that indicate the age of the speaker, even on the telephone. The intensity of vocal symptoms in the elderly and the time of their appearance depend on individual factors and general health conditions. ${ }^{2-4}$ The voice becomes lower, shaky, and breathy; speaking and singing are therefore difficult; and vocal extension decreases. Men present increased fundamental frequency, rendering the voice more acute; women have a deeper voice. ${ }^{4,5}$

Histology and immunohistochemistry studies of elderly larynges identify atrophy of the epithelium, lamina propria, and thyroarytenoid muscle; a decreased concentration of hyaluronic acid and of elastic fibers; and an increased concentration of collagen fibers. ${ }^{6-9}$ Some authors stress that such changes are not only quantitative but also qualitative. ${ }^{9,10}$ For Hammond et al, ${ }^{10}$ the aged larynx presents a decrease in reticular fibers and an increased number of type I collagen fibers, but with impaired function, no longer warranting tissue resistance. The decrease in extracellular matrix glucosamines impairs further vocal fold viscoelasticity, justifying part of the vocal symptoms of the elderly. ${ }^{10}$

Electron microscopy studies of the elderly larynx are scarce yet important, since they may add structural details identifiable by this method. Hirano et $\mathrm{al}^{11}$ found almost twice as many collagen fibers in a study on elderly larynges using scanning electron microscopy, being more evident in the deeper layers of the lamina propria. The authors also found irregular diameters of the collagen fibers and decreased interstitial spaces, separating them from the other elements of the extracellular matrix such as glucosamine and glycosaminoglycans. These authors

\footnotetext{
'Department of Ophthalmology, Otorhinolaryngology and Head and Neck Surgery, Univ Estadual Paulista, Botucatu Medical School (Unesp), Botucatu (SP), Brazil

${ }^{2}$ Institute of Bioscience, Department of Electron Microscopy, Univ Estadual Paulista (Unesp), Botucatu (SP), Brazil
}

\section{Corresponding Author:}

Regina Helena Garcia Martins, MD, PhD, Department of Ophthalmology, Otorhinolaryngology and Head and Neck Surgery, Botucatu Medical School, Univ Estadual Paulista, Distrito de Rubião Junior s/n, I86/8-970, Botucatu, SP, Brazil.

Email: rmartins@fmb.unesp.br 


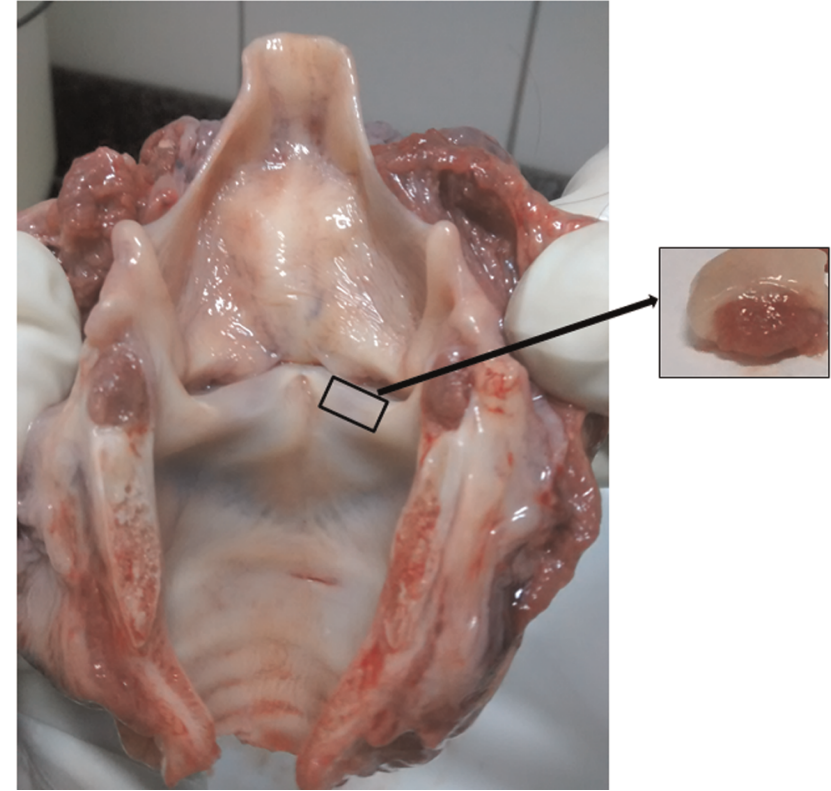

Figure I. Human larynx opened on the posterior portion for macroscopic examination and removal of the right vocal fold.

also noted a decrease in the amount of reticular fibers of the lamina propria, which correspond to a subtype of type III collagen fibers, with diameters of $50 \mathrm{~nm}$. The coexistence and distinction of type I and III collagen fibers in the lamina propria of the vocal folds were also demonstrated by Tateya et $\mathrm{al}^{12}$ in a study with adult human larynges using an interesting technique of immune scanning electron microscopy.

The aging process of the body is complex, and each study on this topic adds valuable information that enhances the understanding on the pathophysiology of the aging process of the many organs and systems. The objective of this study was to describe the findings of scanning electron microscopy (SEM) of vocal folds in the elderly to uncover structural details of the presbylarynx.

\section{Subjects and Methods}

Vocal folds removed from 16 cadavers during necropsy were distributed into 2 age groups: control $(\mathrm{n}=8$; aged 3050 years) and elderly ( $\mathrm{n}=8$; aged $75-92$ years). After harvesting, the larynges were opened on their posterior portion and examined macroscopically to identify lesions, in which case they were excluded.

A cross-sectional fragment of approximately 0.8 to 1.0 $\mathrm{cm}$ of the median portion of the right vocal fold was removed (Figure I). For SEM, the specimens were fixed in $2.5 \%$ glutaraldehyde for 12 hours, flushed in $0.1 \mathrm{M}$ phosphate buffer at $\mathrm{pH} 7.3$, fixed in a $1 \%$ osmium tetroxide solution for 1 hour, flushed in phosphate buffer, dehydrated in increasing solutions of $75 \%$ to $100 \%$ alcohol, and dried in a critical point device (Balzers CPD-020; Balzers AG, Balzers, Liechtenstein) with liquid carbon dioxide. The fragments of the vocal folds were assembled in a metal base with silver glue and then covered with gold (15 nm gold) in a Balzers MED-010 device. They were visualized and
Table I. Thickness of the Epithelium in Both Groups (Mean Values of 10 Measures of Each Larynx).

\begin{tabular}{lll}
\hline & \multicolumn{2}{c}{ Epithelium Thickness, $\mu \mathrm{m}$} \\
\cline { 2 - 3 } Larynx & Elderly Group & Control Group \\
\hline Larynx I & 43.87 & 44.63 \\
Larynx 2 & 37.59 & 46.62 \\
Larynx 3 & 43.47 & 147.17 \\
Larynx 4 & 41.60 & 138.34 \\
Larynx 5 & 40.74 & 140.52 \\
Larynx 6 & 41.02 & 143.23 \\
Larynx 7 & 41.83 & 114.37 \\
Larynx 8 & 44.14 & 176.94 \\
Mean (SD) & $41.79(21.40)$ & $221.64(145.90)$ \\
\hline
\end{tabular}

${ }^{\mathrm{a} P}<.001$.

photographed at increasing magnitudes under a scanning electron microscope (Quanta 200 FEG; FEI Company, Munich, Germany).

To measure the epithelium thickness, we used a scandium morphometric digital program, which measures the distance between 2 points. A straight line was drawn from the distal surface of the epithelium to the beginning of the basal membrane. All measures were analyzed at $400 \times$ magnification, using 10 measures per picture in different sites. The mean values of each picture of both groups are presented in Table I.

We present the details of the epithelium and of the lamina propria components in a descriptive way, comparing the images of the control group with those of the elderly group.

Exclusion criteria included sepsis, prolonged intubation, laryngeal lesions, systemic infectious diseases, dermatological diseases, autoimmune or metabolic disorders, cervical trauma, and respiratory granulomatous diseases such as tuberculosis, paracoccidioidomycosis, sarcoidosis, and other causes or diseases that could affect the vocal fold mucosa and invalidate the study. Medical records were consulted to confirm the predeath diseases, and family members were inquired about the other comorbidities.

The research project was approved by the Human Research Ethics Committee (protocol number 13242413.1.0000.5411) and by the Pathology Department of the same institution.

\section{Statistical Analysis}

The Shapiro-Wilk test was used to determine whether a population followed a normal distribution, with a $P$ value of .11. After that, the Student $t$ test was used to compare the mean values of the thickness, using the $5 \%$ level of significance.

\section{Results}

Scanning electron microscopy indicated that all specimens from the control group were similar. The covering mucosa 


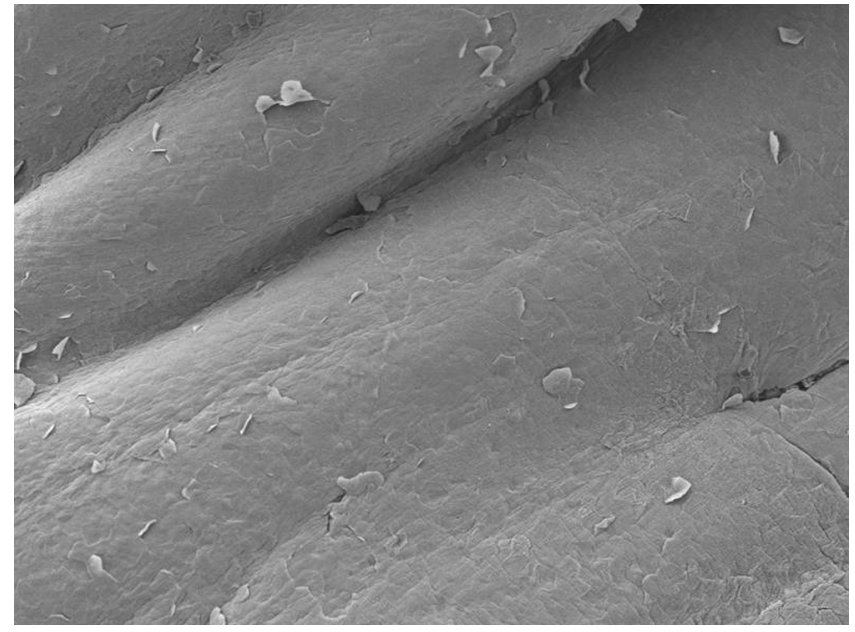

Figure 2. Control group: surface of vocal fold with mild mucosal undulation and some desquamation cells. Scanning electron microscopy, magnification $300 \times$.

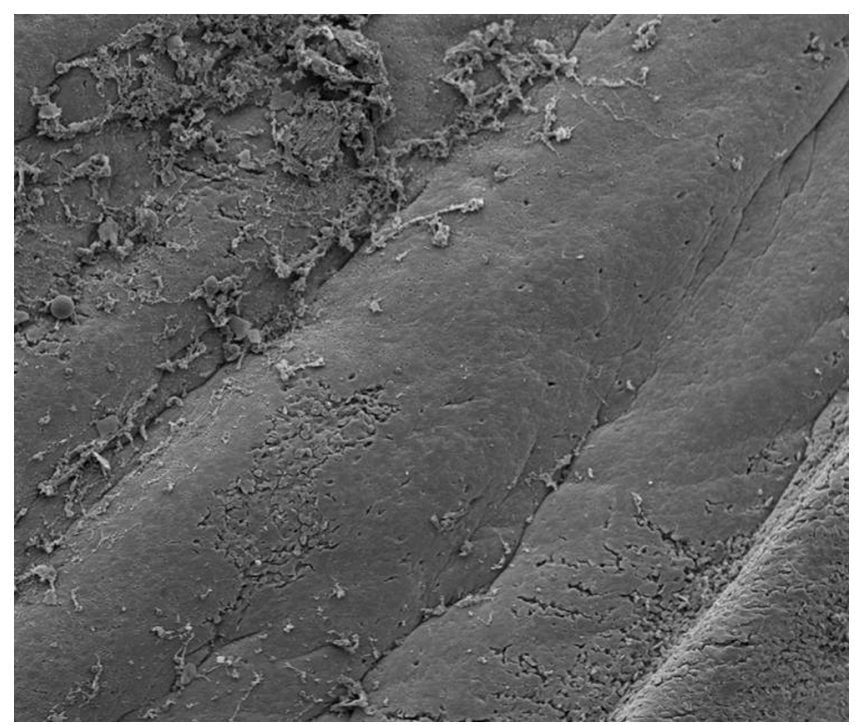

Figure 3. Elderly group: surface of the covering mucosa of the vocal folds more folded and with more desquamation cells. Scanning electron microscopy, magnification $300 \times$.

had soft undulation and few desquamation cells (Figure 2). In the elderly group, most specimens had the covering mucosa more folded and with more desquamation cells (Figure 3). In the control group, microfolds drew a delicate tracery over the surface, and the intercellular junctions demarcated irregular polygons on the surface (Figure 4). In 6 specimens of the elderly group, the intercellular junctions presented deep sulci (Figure 5) and the epithelial surface was irregular.

In the control group, the mucosa was formed by a juxtaposition of 5 to 7 cells that rested over the basal membrane. In the elderly group, the epithelial thickness varied, being normal in 2 specimens (5-7 cells) and atrophic in the other 6 ( $2-3$ cells). The mean (SD) values of the thickness of the epithelium in both groups are presented in Table I

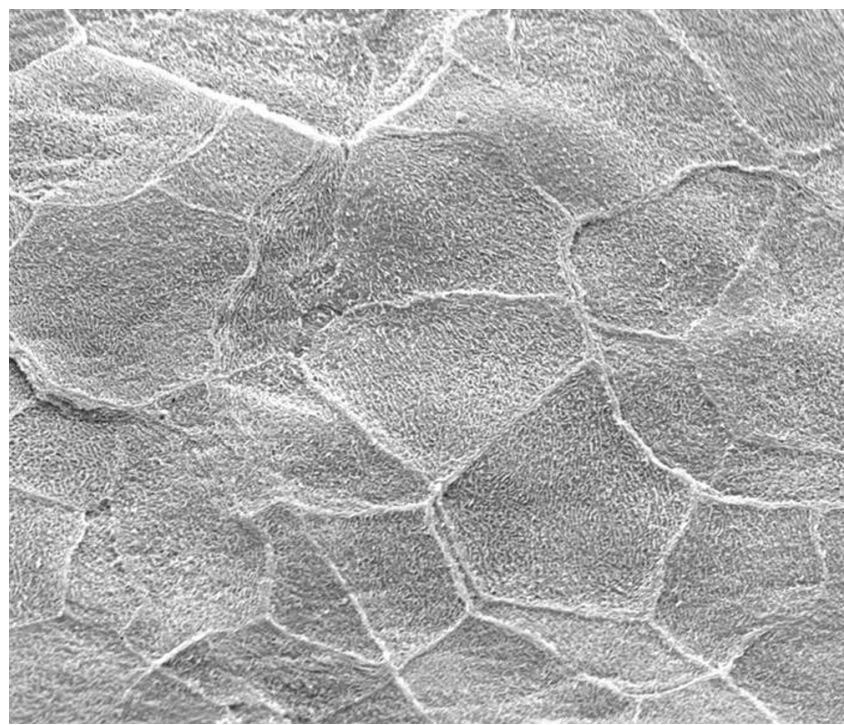

Figure 4. Control group: epithelial surface of vocal fold highlighting the microfolds and protruding intercellular junctions. Scanning electron microscopy, magnification $4000 \times$.

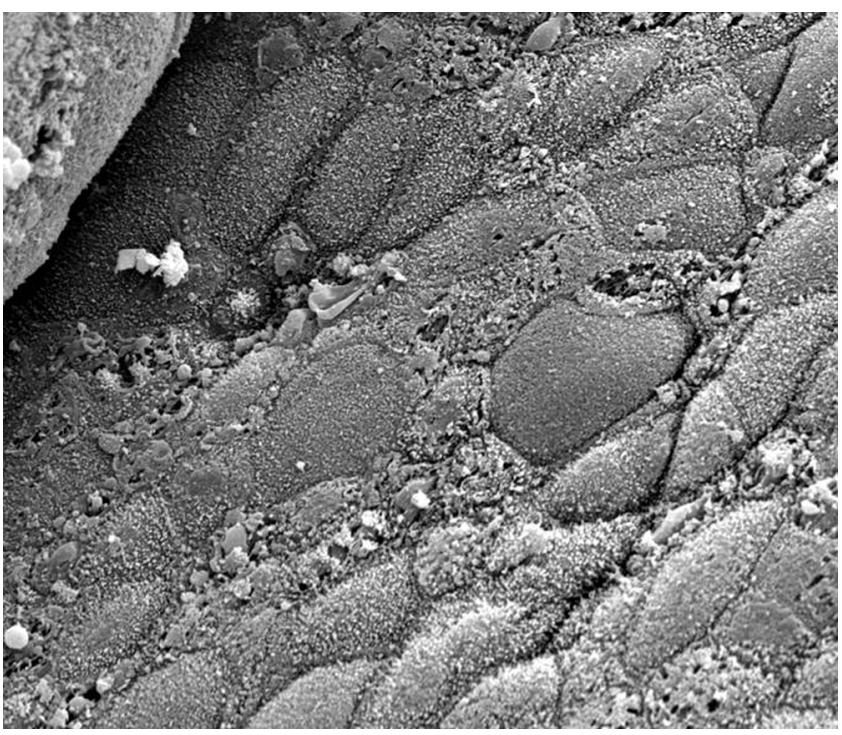

Figure 5. Elderly group: epithelium with well-demarcated cells, separated by deep sulci. Scanning electron microscopy, magnification $4000 \times$.

(control group, 41.79 [21.40] $\mu \mathrm{m}$; elderly group, 221.64 [145.90] $\mu \mathrm{m})$.

In controls, in the superficial layer of the lamina propria, a delicate network of collagen and elastic fibers was seen, running parallel to the basal membrane, just below the epithelium (Figure 6). In the elderly group, in this site, the collagen was more abundant, with irregular distribution (Figure 7). In the deep layer of the lamina propria, there was a dense network of collagen in both groups, but it was more intense in the elderly groups, in which a fibrotic and rigid skeleton could be observed (Figures 8 and 9). 


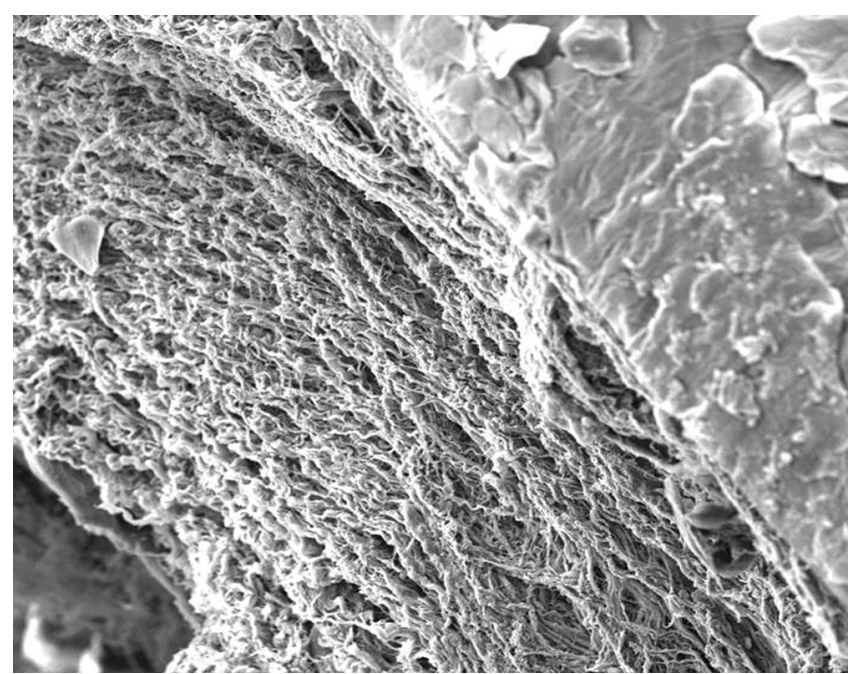

Figure 6. Control group: detail for the loose network of collagen and elastic fibers parallel to the basal membrane in the superficial layer of the lamina propria. Scanning electron microscopy, magnification $600 \times$.

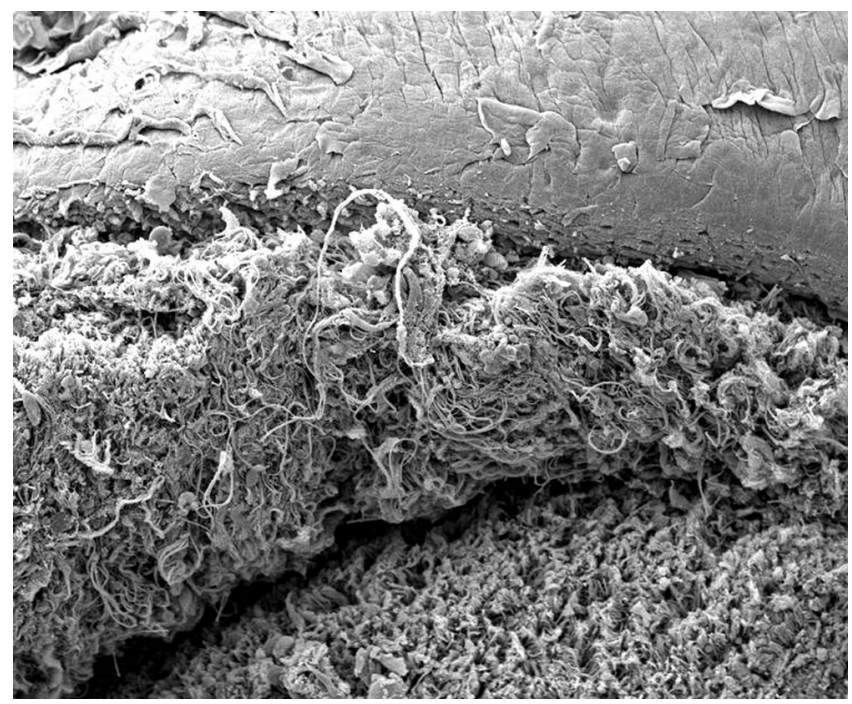

Figure 7. Elderly group: dense and irregular network of collagen and elastic fibers in the superficial layer of the lamina propria. Scanning electron microscopy, magnification $600 \times$.

\section{Discussion}

This study showed several changes in most vocal folds of the elderly, identified by SEM, that have not been reported previously. The limits of the epithelial cells of the vocal folds in the elderly showed deep sulci. The epithelium had an increase of cell desquamation and more undulation. Such changes may be explained by the natural aging process of the epithelium, where the cell junctions may be impaired by the decrease in the components of the extracellular matrix, resulting in laxity, desquamation, and retraction. ${ }^{11,12}$

The absence of studies with electron microscopy of the older larynx renders impossible the comparison of our

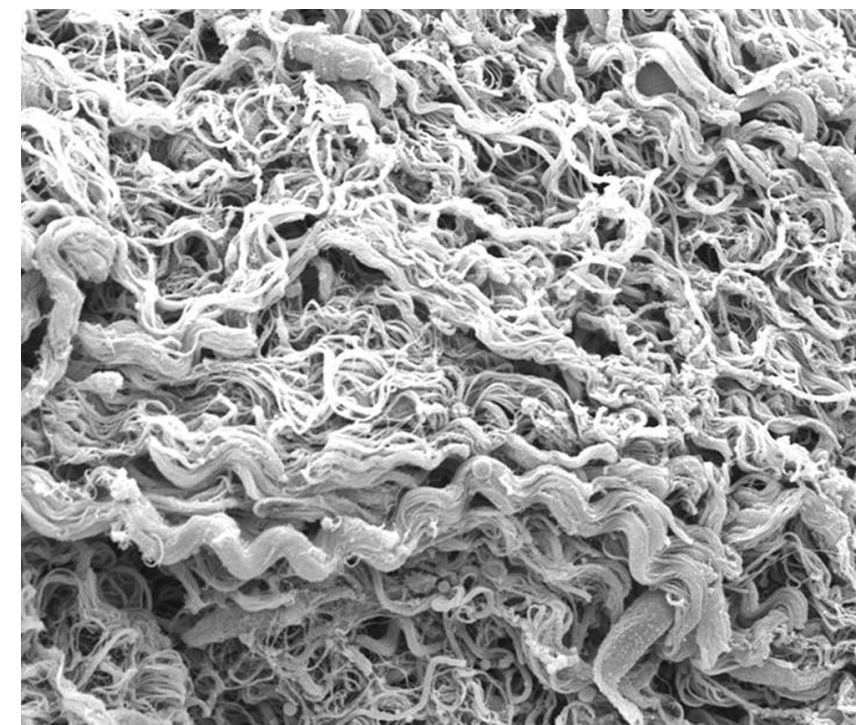

Figure 8. Control group: detail for the dense network of collagen in the deeper layer of the lamina propria. Scanning electron microscopy, magnification $2400 \times$.

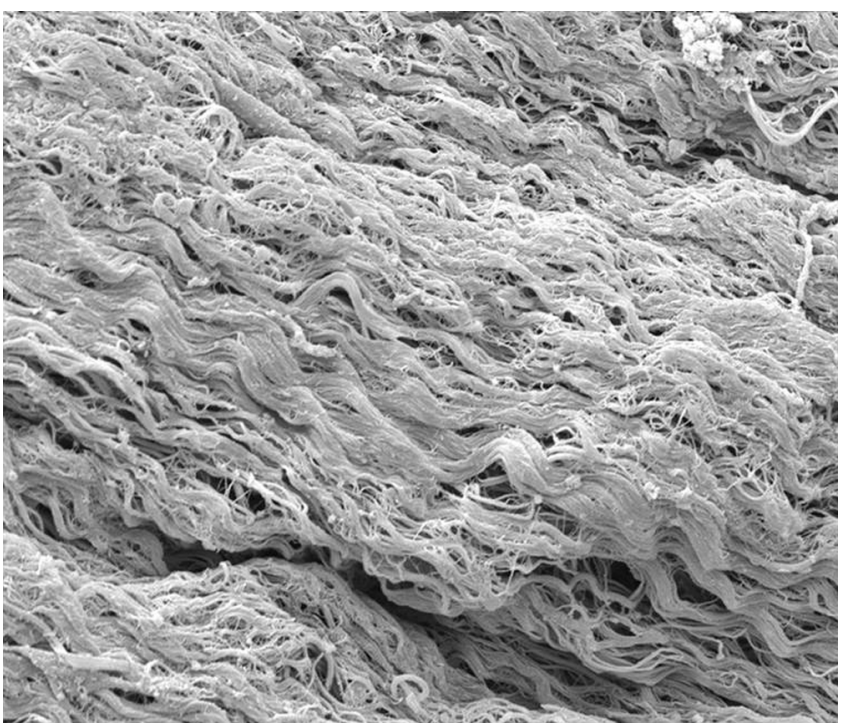

Figure 9. Elderly group: detail for the collagen more abundant in the deeper layer of the lamina propria, forming a fibrotic and rigid skeleton. Scanning electron microscopy, magnification $2400 \times$.

descriptions with that of other authors. There are, nevertheless, many studies describing the skin aging process, with the epithelium being similar, and therefore correlations are possible. When identified in the epidermis, such changes have been attributed to the loss of fundamental substances of the extracellular matrix, causing wrinkles, epidermal retractions, loss of the proliferative capacity of the epithelium, and frailty. ${ }^{13,14}$ The dermal lamina propria is described also as compact, dense, and made of collagens and elastic fibers, many of which degenerate, reinforcing the hypothesis that the changes in the proteins of the lamina propria are not only quantitative but also qualitative. 
The behavior of the elastic fibers in the vocal folds of the elderly has been discussed by authors. Some authors found an important decrease in their concentration, others considered the amount unaltered, and others yet found a considerable increase. ${ }^{10-12,15}$ Hammond et al ${ }^{10}$ analyzed the concentration of elastic fibers in the lamina propria of 19 larynges, including children, adults, and the elderly, and found a growing increase in elastin as age advances, indicating maturity of the structures of the vocal folds. The authors also observed increasing growth in collagen fibers and underscored that this result not necessarily indicated increased production but rather a decrease in turnover. Roberts et $\mathrm{al}^{15}$ supported this same hypothesis and described the irregular distribution and larger number of collagen fibers in the lamina propria of the vocal folds of the elderly, ratifying our findings, as did other authors. ${ }^{6-9}$ Those authors indicate that the collagen fibers do not finish degradation as a consequence of impairing the expression of the enzyme collagenase. The mature collagen fibers that are not degraded become distorted, forming irregular arrangements and clustering in an entanglement of fibers with different diameters and in all directions, impairing tissue flexibility and elasticity. The deeper layers of the lamina propria receive a higher concentration of collagen fibers, as depicted in Figure 9, forming a rigid skeleton. In our study, unfortunately it was not possible to differentiate the elastic fibers of the reticular collagen, requiring additional methods for this purpose.

Structural changes in the fibroblasts are yet little known and seem to be responsible for the modifications in the proteins of the extracellular matrix of the vocal folds of the elderly. Hirano et $\mathrm{al}^{11}$ believe it is important to study the behavior of those cells, due to their key participation in the production of the elements of the extracellular matrix. In an interesting research study, those authors studied the fibroblasts of human larynges in neonates, adults, and older persons and found that the shape of those cells changed as age progressed, going from oval in neonates to oblong and filamentous in adults and older persons. Furthermore, they emphasized that active fibroblasts had well-developed organelles and a specially undulated endoplasmic reticulum and Golgi complex, which tend to decrease in the elderly.

Quan et $\mathrm{al}^{14}$ in a study on skin fibroblasts in the elderly, identified many fibroblasts with a deletion in the mitochondrial DNA, impairing modulation of the fibroblasts and the configuration of the actin filaments of the cytoskeleton. According to those authors, such deletions might be triggered by oxidative stress and mitigated with the introduction of antioxidants.

For Roberts et $\mathrm{al},{ }^{15}$ part of the changes observed in the aged vocal folds can be due to the reduction in the activity of fibroblasts, as a consequence of the possible decrease in blood flow. However, the impairment in the vascular network of vocal folds of elderly larynxes could not be confirmed by these authors.

\section{Conclusion}

Scanning electron microscopy identified several changes in the elderly larynx, differentiating it from the controls. These alterations are probably related to the aging process of the vocal folds. However, the exact interpretation of these findings requires additional studies, even to the molecular level, having the fibroblasts as targets.

\section{Acknowledgment}

To the Fapesp for the financial support.

\section{Author Contributions}

Tatiana Maria Gonçalves, conception of the work, drafting the work, final approval of the version to be published, data analysis; Daniela Carvalho dos Santos, final approval of the version to be published, drafting the work, conception or design of the work, drafting the work and revising it critically; Adriana Bueno Benito Pessin, conception of the work, drafting the work, final approval of the version to be published, data analysis; Regina Helena Garcia Martins, conception or design of the work, drafting the work and revising it critically, final approval of the version to be published, data analysis.

\section{Disclosures}

Competing interests: None.

Sponsorships: Fapesp (Fundação de Amparo à Pesquisa do Estado de São Paulo) and CNPq (Conselho Nacional de Desenvolvimento Científico e Tecnológico), for collection, analysis, and interpretation of the data.

Funding source: Fapesp (Fundação de Amparo à Pesquisa do Estado de São Paulo) and CNPq (Conselho Nacional de Desenvolvimento Científico e Tecnológico), for collection, analysis, and interpretation of the data, as well as financial support for histological analysis.

\section{References}

1. Kendall K. Presbyphonia: a review. Curr Opin Otolaryngol Head Neck Surg. 2007;15:137-140.

2. Takano S, Kimura M, Nito T, Imagawa H, Sakakibara K, Tayama N. Clinical analysis of presbylarynx — vocal fold atrophy in elderly individuals. Auris Nasus Larynx. 2010;37:461-464.

3. Gregory ND, Chandran S, Lurie D, Sataloff RT. Voice disorders in the elderly. $J$ Voice. 2012;26:254-258.

4. Martins RH, Gonçalves TM, Pessin ABB, Branco A. Aging voice: presbyphonia. Aging Clin Exp Res. 2014;26:1-5.

5. Pontes P, Brasoloto A, Behlau M. Glottic characteristics and voice complaint in the elderly. J Voice. 2005;19:84-94.

6. Sato K, Hirano M, Nakashima T. Age-related changes of collagenous. Ann Otol Rhinol Laryngol. 2002;111:15-20.

7. Ximenes Filho JA, Tsuji DH, Nascimento PHS, Sennes LU. Histologic changes in human vocal folds correlated with aging: a histomorphometric study. Ann Otol Rhinol Laryngol. 2003;112:894-898.

8. Ohno T, Hirano S, Rousseau B. Age-associated changes in the expression and deposition of vocal fold collagen and hyaluronan. Ann Otol Rhinol Laryngol. 2009;118:735-741. 
9. Pontes P, Yamasaki R, Behlau M. Morphological and functional aspects of the senile larynx. Folia Phoniatr Logop. 2006;58:151-158.

10. Hammond T, Gray S, Butler J. Age and gender related collagen distributions in human vocal folds. Ann Otol Rhinol Laryngol. 2000;109:913-920.

11. Hirano M, Sato K, Nakashima T. Fibroblasts in geriatric vocal fold mucosa. Acta Otolaryngol. 2000;120:336-340.

12. Takeda N, Thomas GR, Ludlow CL. Aging effects on motor units in the human thyroarytenoid muscle. Laryngoscope. 2000;110:1018-1025.
13. Lavker RM, Zheng PS, Dong G. Morphology of aged skin. Clin Geriatr Med. 1989;5:53-67.

14. Quan C, Cho MK, Perry D, Quan T. Age-associated reduction of cell spreading induces mitochondrial DNA common deletion by oxidative stress in human skin dermal fibroblasts: implication for human skin connective tissue aging. $J$ Biomed Sci. 2015;28;22:62.

15. Roberts T, Morton R, Ali-Ali S. Microstructure of the vocal fold in elderly humans. Clin Anat. 2011;24:544-551. 